\title{
PROTOCOLO DE DETECÇÃO, DIAGNÓSTICO E TRATAMENTO DO DIABETES MELLITUS, NA GRAVIDEZ
}

PROTOCOL FOR DETECTION, DIAGNOSIS AND TREATMENT OF DIABETES MELLITUS DURING PREGNANCY

Renan M. Montenegro Jr. ${ }^{1}$; Glória Maria G. F. Paccola²; Milton Cesar Foss ${ }^{3}$; Maria Tereza C.G. Torquato4; Rafael K. Yano ${ }^{5}$; Francisco Mauad Filho ${ }^{6}$; Antonio Alberto Nogueira ${ }^{6}$; Aderson Tadeu Berezowski ${ }^{6}$ \& Geraldo Duarte ${ }^{6}$

${ }^{1}$ Médico Pós-graduando, ${ }^{2}$ Médica Assistente, ${ }^{3}$ Docente, do Departamento de Clínica Médica da Faculdade de Medicina de Ribeirão Preto-USP. ${ }^{4}$ Médica Assistente, Doutora, Coordenadora do Programa de Diabetes da Secretaria de Saúde de Ribeirão Preto. ${ }^{5}$ Médico Assistente, ${ }^{6}$ Docente, do Departamento de Ginecologia e Obstetrícia da Faculdade de Medicina de Ribeirão Preto -USP.

Correspondência: Milton Cesar Foss - Departamento de Clínica Médica da Faculdade de Medicina de Ribeirão Preto - USP - Avenida Bandeirantes, 3900 - Ribeirão Preto, São Paulo. CEP: 14049-900 - e-mail:mcfoss@fmrp.usp

MONTENEGRO Jr. RM; PACCOLA GMGF; FOSS MC; TORQUATO MTCG; YANO RK; MAUAD FILHO F; NOGUEIRA AA; BEREZOWSKI AT \& DUARTE G. Protocolo de detecção, diagnóstico e tratamento do Diabetes mellitus na gravidez. Medicina, Ribeirão Preto, 33: 520-527, out.dez. 2000.

RESUMO: Os autores apresentam a abordagem da paciente gestante, para rastreamento e diagnóstico do Diabetes mellitus Gestacional (DMG) e tratamento do Diabetes mellitus durante a gravidez, estabelecida como procedimento rotineiro pelo Setor de Gestação de Alto Risco do Departamento de Ginecologia e Obstetrícia e Divisão de Endocrinologia e Metabologia do Departamento de Clínica Médica da Faculdade de Medicina de Ribeirão Preto - Universidade de São Paulo e adotado na avaliação pré-natal da Secretaria Municipal de Saúde de Ribeirão Preto para rastreamento e diagnóstico do DMG. Este protocolo foi baseado nas recomendações da Organização Mundial da Saúde e American Diabetes Association.

UNITERMOS: Gravidez no Diabetes. Diabetes Gestacional . Diagnóstico. Protocolos Clínicos.

\section{INTRODUÇÃO}

A gestação em mulheres diabéticas é uma condição reconhecidamente associada a uma maior freqüência de anormalidades, quando comparada a gestações normais. Sabe-se que a hiperglicemia, nesse período, pode resultar em aumento da mortalidade fetal, além de uma maior frequiência de complicações tais como malformações, macrossomia, hipoglicemia, hiperbilirrubinemia, policitemia, hipocalcemia, hipomagnesemia, cardiomiopatia hipertrófica e síndrome do desconforto respiratório do recém-nascido. Para a gestante, o mau controle metabólico está implicado em maiores índices de abortos espontâneos, infecções, hipertensão arterial, doença hipertensiva específica da gravidez (DHEG), partos pré-termo e cesáreas. Embora ainda não estejam completamente definidos os níveis ideais de glicemia durante a gestação nas pacientes diabéticas, já está demonstrado que um bom controle metabólico está associado à redução dessas complicações $^{(1 / 6)}$.

A gravidez é uma condição fisiológica em que ocorre uma adaptação hormonal, representada por aumento dos níveis de estrógenos, progesterona, cortisol, prolactina e produção de lactogênio placentário humano. Essas mudanças interferem no metabo- 
lismo dos carboidratos, podendo resultar, em mulheres susceptíveis, no desencadeamento de DMG e, naquelas previamente diabéticas, em piora do controle glicêmico.

Segundo a American Diabetes Association(7), a classificação do Diabetes mellitus (DM) pode ser resumida em quatro grandes grupos, como apresentado na Tabela I. Pacientes com qualquer forma de diabetes podem necessitar de insulinoterapia em algum estágio da sua doença. Portanto, nas pacientes com diagnóstico anterior à gestação, a utilização de insulina não define o tipo clínico do diabetes, sendo necessária a observância de outros critérios clínicos (ocorrência prévia de cetoacidose, história familiar de DM, presença de obesidade, acanthosis nigricans ou outras patologias associadas).

\section{Tabela I - Classificação do Diabetes mellitus}

\section{- Diabetes tipo 1}

A. Auto-imune

B. Idiopático

\section{- Diabetes tipo 2}

\section{- Outros tipos específicos}

A. Defeitos genéticos da função da célula $\beta$

B. Defeitos genéticos da ação da insulina

C. Doenças do pâncreas exócrino

D. Endocrinopatias

E. Induzido por drogas ou substâncias químicas

F. Infecções

G. Formas incomuns de diabetes imune

$\mathrm{H}$. Outras síndromes genéticas associadas ao diabetes

\section{- Diabetes gestacional}

O Diabetes mellitus gestacional (DMG) é definido como intolerância à glicose de intensidade variável, detectada, pela primeira vez, durante a gestação ${ }^{(8)}$. Esse distúrbio é uma das complicações mais comuns da gestação, com uma prevalência de 2 a $15 \%$, dependendo da população estudada e dos critérios diagnósticos utilizados.

A despeito da hiperglicemia ser de intensidade leve, na maioria dos casos, o DMG mal controlado também resulta numa elevada morbidade materna e fetal $^{(1,2,9,10)}$. Além disso, outra importante implicação do DMG é o maior risco materno de desenvolvimento de intolerância à glicose ou DM após a gestação ${ }^{(11,12,13)}$.

Considerando que a apresentação do DMG é geralmente assintomática, e sabendo-se que a terapêutica adequada pode reduzir a freqüência de complicações, torna-se imperativa a avaliação de todas as gestantes, iniciando-se na primeira consulta pré-natal ${ }^{(14,15)}$.

No presente trabalho, apresentamos a abordagem da gestante diabética, baseada nas recomendações da Organização Mundial da Saúde ${ }^{(16)}$ e American Diabetes Association ${ }^{(8)}$, estabelecida como procedimento rotineiro pelo Setor de Gestação de Alto Risco do Departamento de Ginecologia e Obstetrícia e Divisão de Endocrinologia e Metabologia do Departamento de Clínica Médica da FMRP-USP e adotado na avaliação pré-natal da Secretaria Municipal de Saúde de Ribeirão Preto para rastreamento e diagnóstico do DMG.

\section{RASTREAMENTO}

A proposta deste protocolo é a adoção do rastreamento universal, ou seja, todas as gestantes devem ser submetidas à avaliação, realizada já na primeira consulta pré-natal e reavaliação posterior (entre a $24^{\mathrm{a}}$ e $28^{\mathrm{a}}$ semana), se o resultado inicial for negativo.

Apesar de utilizada em outras populações, a realização de rastreamento para DMG, somente em gestantes de risco, não nos parece adequada, por não ter sido validada no nosso meio e devido às características étnicas da nossa população. Os fatores considerados de risco para DMG pela American Diabetes Association $^{(7,17)}$ são: idade maior de 25 anos, obesidade, história familiar de Diabetes mellitus, origem hispânica, afro-americana, asiática ou nativa americana, história obstétrica ruim e tolerância à glicose previamente anormal. Com a utilização desse tipo de rastreamento no nosso meio, existe a possibilidade de não detecção de casos positivos para essa complicação ${ }^{(18)}$. Como pode ser notado, seguindo-se os citados critérios, a grande maioria da população brasileira seria considerada de risco para essa doença. Fica claro que os critérios não são adequados para a nossa realidade, sendo necessários estudos objetivos para determinar quais são os fatores de risco específicos para a nossa população.

$\mathrm{O}$ rastreamento consiste, inicialmente, no teste oral de tolerância à glicose com 50 gramas (TOTG-50), e medidas de glicemia plasmática, venosa, de jejum e de $1 \mathrm{~h}$ após a sobrecarga. A medida da 


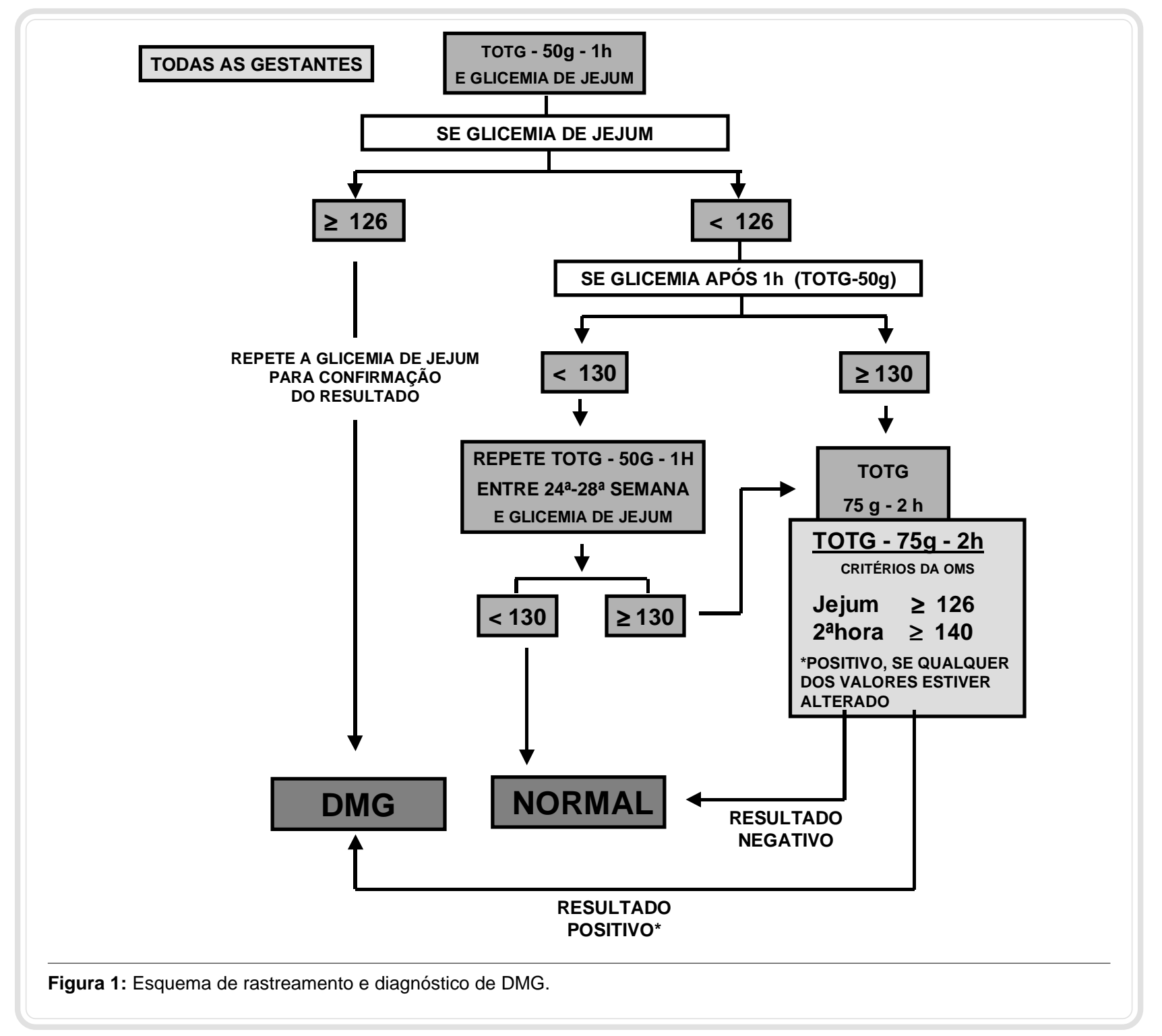

glicemia plasmática venosa, em jejum, servirá para identificação de casos assintomáticos de Diabetes mellitus prévio (Figura 1). Um resultado de glicemia de jejum maior ou igual a $126 \mathrm{mg} / \mathrm{dl}$, confirmado com repetição do exame, é considerado como diagnóstico de DMG. Caso contrário, deve ser analisada a glicemia de $1 \mathrm{~h}$ do TOTG-50. Considera-se rastreamento positivo, quando a glicemia de $1 \mathrm{~h}$ após o TOTG-50 for maior ou igual a $130 \mathrm{mg} / \mathrm{dl}$, estando indicado o teste de tolerância à glicose com 75 gramas para diagnóstico (item III). Se a glicemia de $1 \mathrm{~h}$, no teste com 50 gramas, for menor que $130 \mathrm{mg} / \mathrm{dl}$, considera-se rastreamento negativo, devendo ser repetido entre a $24^{\mathrm{a}}$ e $28^{\mathrm{a}}$ semana e analisado, utilizando-se os mesmos critérios.

\section{DIAGNÓSTICO}

O diagnóstico é baseado nas determinações da Organização Mundial da Saúde ${ }^{(16)}$, que preconiza o teste de tolerância à glicose via oral com 75 gramas (TOTG-75), com medida das glicemias plasmáticas venosas de jejum e 2h. Considera-se diagnóstico de DMG, se a glicemia de jejum for maior ou igual a $126 \mathrm{mg} / \mathrm{dl}$ ou a de 2 h (TOTG-75) maior ou igual a $140 \mathrm{mg} / \mathrm{dl}$. Na gestante, cujo rastreamento ou avaliação diagnóstica for negativa entre a 24 e 28 semanas, e que apresente um quadro sugestivo de DMG (ganho de peso acentuado, macrossomia ou poliidrâmnio na avaliação ultrasonográfica), deve-se considerar uma nova reavaliação com 32 semanas com um TOTG-75. 
Para execução e interpretação correta do teste de tolerância à glicose via oral com 75 gramas (TOTG75), as seguintes recomendações devem ser seguidas: 1) o procedimento deve ser realizado pela manhã, com a gestante em jejum prévio de 8 a $14 \mathrm{~h}$, sem restrições alimentares nos três dias anteriores ao teste (mínimo de 150 gramas de carboidratos por dia) e da atividade física usual; 2) após a coleta da amostra em jejum, a paciente ingere 75 gramas de glicose anidra ou equivalente, dissolvidas em $300 \mathrm{ml}$ de água, em período de tempo não superior a $5 \mathrm{~min}$; 3 ) durante o teste, a gestante deve manter-se em repouso e sem fumar; o exame não deve ser realizado em vigência de infecções ou outras doenças intercorrentes; as medicações em uso devem ser anotadas; 4) as amostras de sangue devem ser centrifugadas em até $2 \mathrm{~h}$ após cada coleta ou conservadas com fluoreto de sódio (6 mg para cada $1 \mathrm{ml}$ de sangue) e mantidas em freezer; deve ser evitada hemólise.

\section{AVALIAÇÃO DE COMPLICAÇÕES CRÔ- NICAS E PATOLOGIAS ASSOCIADAS AO DIABETES}

Para avaliar possíveis complicações relacionadas ao Diabetes mellitus, devem ser solicitadas, ao diagnóstico e a cada trimestre os exames listados na Tabela II.

\section{TRATAMENTO}

O esquema terapêutico na paciente com DMG tem três pontos fundamentais: monitorização glicêmica, orientação nutricional e insulinoterapia. Essa fica res-

\begin{tabular}{|l|}
\hline $\begin{array}{l}\text { Tabela II - Avaliação de complicações crônicas e } \\
\text { patologias associadas ao diabetes. }\end{array}$ \\
\hline - Hemoglobina glicosilada $\left(\mathrm{HbA}_{1 \mathrm{c}}\right)$ \\
- Proteínas totais e albumina \\
- Proteinúria de 24 h ou microalbuminúria \\
- Lipidograma \\
- Uréia e creatinina \\
- Sódio, potássio e cálcio séricos \\
- Ácido úrico \\
- Urina rotina \\
- Urocultura \\
- Fundoscopia \\
- ECG \\
\hline * Os dois últimos, somente ao diagnóstico do DMG. \\
\hline
\end{tabular}

trita a um menor número de casos, em que não se obtém o controle glicêmico adequado com a dietoterapia isoladamente. Os antidiabéticos orais não devem ser utilizados durante a gestação ${ }^{(19,20)}$.

\subsection{Monitorização glicêmica}

O controle metabólico é realizado com medidas de glicose plasmática em sangue venoso ou glicemias capilares por glicosímetro. A medida de glicosúria não é adequada.

As glicemias plasmáticas venosas são realizadas durante as internações e a cada retorno ao ambulatório. São colhidas amostras às $7 \mathrm{~h}$ (em jejum), 10, 14,20 e $2 \mathrm{~h}$ da madrugada. As duas últimas são realizadas apenas durante internações. No caso de disponibilidade de glicosímetro, é recomendada a monitorização domiciliar diária, com medidas de glicemia capilar até quatro vezes por dia (em jejum, pré e pósprandiais), de acordo com a condição para utilização das fitas.

Os parâmetros de bom controle são (1): glicemia de jejum menor que $90 \mathrm{mg} / \mathrm{dl}$ (sangue venoso) ou menor que $95 \mathrm{mg} / \mathrm{dl}$ (amostra capilar); glicemias pósprandiais de $1 \mathrm{~h}$, menores que $140 \mathrm{mg} / \mathrm{dl}$, e de $2 \mathrm{~h}$, menores que $120 \mathrm{mg} / \mathrm{dl}$. O limite inferior para qualquer horário é de $60 \mathrm{mg} / \mathrm{dl}$. Havendo glicemias maiores que $200 \mathrm{mg} / \mathrm{dl}$ ou menores que $50 \mathrm{mg} / \mathrm{dl}$, está indicada internação (Tabela III).

Os retornos devem ser a cada duas semanas até a $32^{\mathrm{a}}$ semana da gestação. Após isso, os retornos devem ser semanais. Deve-se avaliar a glicemia das $2 \mathrm{~h}$ da madrugada pelo menos uma vez por mês.

\subsection{Dieta}

A intervenção nutricional é o aspecto mais importante do tratamento da paciente com diabetes mellitus gestacional.

Diagnosticado o DMG, a terapêutica inicial consiste, na quase totalidade dos casos, em dietoterapia exclusiva, que deve ser individualizada e mantida

Tabela III - Parâmetros de controle glicêmico.

$$
\begin{array}{ll}
\text { Glicemia de jejum }(7 \mathrm{~h}) & <95 \mathrm{mg} / \mathrm{dl}^{\star} \\
\text { Glicemia às } 10 \mathrm{~h} & <120 \mathrm{mg} / \mathrm{dl} \\
\text { Glicemias pós-prandiais }(14 \text { e } 20 \mathrm{~h}) & <120 \mathrm{mg} / \mathrm{dl} \\
\text { Glicemia noturna }(2 \mathrm{~h}) & >60 \mathrm{mg} / \mathrm{dl}
\end{array}
$$

* $<90 \mathrm{mg} / \mathrm{dl}$ se glicemia de jejum em amostra de sangue venoso 
durante uma a duas semanas. Ela deve se adequar às necessidades e características individuais daquela gestante.

O ganho de peso ideal, durante a gestação, é, geralmente, inversamente proporcional ao peso antes da concepção. Com isso, é necessário determinar o índice de massa corpórea (IMC) nessa fase e estabelecer os limites quanto à evolução ponderal da gestante. Nas pacientes obesas (IMC $\left.>29 \mathrm{~kg} / \mathrm{m}^{2}\right)^{(8)}$. Naquelas com baixo peso (IMC $<19,8 \mathrm{~kg} / \mathrm{m}^{2}$ ) é permitido um ganho ponderal de até $18 \mathrm{~kg}$. Nas pacientes que, antes da gravidez, tinham o peso adequado ou sobrepeso, o incremento deverá ficar entre esses limites, sendo inversamente proporcional ao IMC. Semanalmente, o ganho ponderal deve ser, em média, de 230 a 450 gramas. Ao avaliar o aumento do peso, considerar sempre outros fatores, como edema de membros inferiores, presença de macrossomia ou poliidrâmnio.

Qualitativamente, a dieta deve ser composta de $35-40 \%$ de carboidratos, 20-25\% de proteínas e 35$40 \%$ de lipídios. Devido aos seus efeitos sobre a glicemia pós-prandial, os carboidratos simples devem ser evitados. O cálculo calórico diário total deve ficar entre 25 e $40 \mathrm{kcal} / \mathrm{kg}$, de acordo com o IMC. Devido ao risco aumentado de cetonemia, dietas com menos de $25 \mathrm{kcal} /$ dia só devem ser utilizadas em situações especiais, como em pacientes com obesidade mórbida, havendo necessidade de monitorização. A restrição calórica, mesmo nas pacientes mais obesas, deve ser instituída de maneira cautelosa, dada a maior susceptibilidade de gestantes diabéticas à desnutrição protéica. Essa situação tem sido implicada no desenvolvimento de macrossomia e ocorrência futura de diabetes mellitus do tipo 2, no feto ${ }^{(21,22)}$.

\subsection{Insulinoterapia}

Avaliando o controle glicêmico e adesão à dietoterapia, através do recordatório alimentar e da evolução ponderal, será definida a necessidade de insulinoterapia. Na gestação, recomenda-se utilizar insulinas humanas. A dose total diária varia de 0,8 a 2,0 U/kg.

Inicia-se com uma insulina de ação intermediária (NPH ou lenta) em dose única matinal $(0,3 \mathrm{U} / \mathrm{kg} /$ dia). Em casos específicos, como naqueles em que somente a glicemia de jejum está elevada, pode-se iniciar a insulinização com uma dose de ação intermediária, ao deitar $(0.1 \mathrm{U} / \mathrm{kg} / \mathrm{dia})$.

A adequação do esquema é feita, levando em conta o controle glicêmico e ganho de peso. A depender desses parâmetros, são feitos os ajustes, poden- do-se utilizar doses múltiplas de insulina de ação intermediária, associadas ou não às de ação rápida. $\mathrm{O}$ esquema convencional consiste em uma a duas injeções diárias de insulina de ação intermediária, uma antes do café da manhã e uma antes do jantar. Eventualmente, se as glicemias das 10 e $20 \mathrm{~h}$ se mantiverem elevadas, pode ser acrescentada insulina de ação rápida nesses horários. Se ocorrer hipoglicemia noturna, a dose da insulina de ação intermediária do jantar deve ser diminuída ou administrada ao deitar.

Em esquemas mais intensivos, utilizam-se múltiplas doses de insulina de ação rápida antes das refeições, além das de ação intermediária ou prolongada. Para isso, levam-se em conta, além do controle metabólico, as condições socioeconômicas e a motivação da paciente.

Durante o seguimento, o aumento da dose de insulina deve ser de aproximadamente $20 \%$ da dose anterior a cada retorno, levando-se em consideração o ganho ponderal, o perfil glicêmico e a resposta a um aumento de dose anterior.

\subsection{Atividade física}

A atividade física deve ser estimulada na paciente com DMG. Considera-se segura, quando não é extenuante, não causa estresse fetal ou contrações uterinas.

As pacientes que já praticavam exercícios físicos antes da gestação, devem continuar a praticá-los. Naquelas sedentárias, obesas ou mais idosas recomendam-se exercícios aeróbicos, como caminhadas e hidroginástica, com início gradual e, se possível, supervisionados.

\section{INTERNAÇÃO}

Além das indicações obstétricas, a internação deverá ocorrer quando houver glicemias maiores que $200 \mathrm{mg} / \mathrm{dl}$ ou menores que $50 \mathrm{mg} / \mathrm{dl}$. Também está indicada a internação quando não estiver sendo obtido controle adequado com a orientação nutricional e ajuste da insulinoterapia.

Os objetivos são semelhantes aos do acompanhamento ambulatorial.

Em caso de internação por mau controle glicêmico, mantém-se a dose que vinha sendo utilizada anteriormente em ambulatório, reorienta-se a dieta e reavalia-se com perfil glicêmico $(7,10,14,20$ e 2 h.) após dois a três dias.

O ajuste das doses deve ser feito de modo semelhante ao utilizado no seguimento ambulatorial. 


\section{O PARTO}

A ocorrência de diabetes gestacional não determina a necessidade de parto cesárea. A via do parto é de indicação obstétrica.

No dia do parto, a glicemia deve ser mantida entre 80 e $120 \mathrm{mg} / \mathrm{dl}$ para medidas em sangue venoso e entre 70 e $110 \mathrm{mg} / \mathrm{dl}$ para amostra capilar ${ }^{(8)}$.

\subsection{Parto eletivo - cesárea}

O parto deve ser realizado de preferência pela manhã. Nesse caso, mantém-se a dieta e o esquema de insulina que vinha sendo utilizado até o dia anterior. Após a meia-noite, à gestante é orientado jejum. No dia do parto, não deve ser administrada insulina de ação intermediária. Deixa-se uma infusão de uma solução de glicose a $10 \%$, visando fornecer 150 a $200 \mathrm{~g}$ de glicose/dia. Monitoriza-se a glicemia (capilar ou venosa) de $1 \mathrm{em} 1 \mathrm{~h}$ e administra-se insulina regular conforme glicemia (Tabela IV). A infusão da solução com glicose deve ser aumentada, caso se atinjam níveis de glicemia inferiores aos limites desejados $(<70 \mathrm{mg} / \mathrm{dl}$ em glicemia capilar ou $80 \mathrm{mg} / \mathrm{dl}$ para sangue venoso).

Tabela IV - Esquema de administração de insulina durante o parto.

$\begin{array}{ll}\text { - até } 120 \mathrm{mg} / \mathrm{dl} & 0 \\ -120-160 \mathrm{mg} / \mathrm{dl} & 4 \text { unidades } \\ -161-200 \mathrm{mg} / \mathrm{dl} & 6 \text { unidades } \\ ->200 \mathrm{mg} / \mathrm{dl} & 8 \text { unidades }\end{array}$

\subsection{Parto normal e urgências}

Suspender, se possível, a última dose de insulina de ação intermediária. Iniciar esquema insulínico semelhante ao do parto eletivo.

\subsection{Cuidados neonatais}

Os partos devem ser acompanhados por pediatra da Unidade de Neonatologia com especial atenção para as anormalidades fetais relacionadas ao diabetes. Macrossomia, malformações, prematuridade, hipoglicemia, síndrome do desconforto respiratório do recém-nascido, hipocalcemia, hipomagnesemia, hiperbilirrubinemia e óbito fetal são complicações descritas com frequiência aumentada em gestantes diabéticas $^{(1 / 6)}$.

\section{PUERPÉRIO}

As pacientes com DMG raramente necessitam de insulina exógena após o parto.

\subsection{Imediato}

De qualquer forma, deve-se fazer a monitorização glicêmica no período imediatamente após o parto, inicialmente de $4 \mathrm{em} 4 \mathrm{~h}$, com aumento dos intervalos ou interrupção de acordo com o controle. Se necessário, fazer insulina de ação rápida conforme glicemia (Tabela V).

\begin{tabular}{|c|c|}
\hline - até $200 \mathrm{mg} / \mathrm{dl}$ & 0 \\
\hline $201-250 \mathrm{mg} / \mathrm{dl}$ & 4 unidades \\
\hline - $251-300 \mathrm{mg} / \mathrm{dl}$ & 8 unidades \\
\hline$->300 \mathrm{mg} / \mathrm{dl}$ & 12 unidades \\
\hline
\end{tabular}

\subsection{Tardio}

Após a normalização no pós-parto imediato, manter sem insulina até o retorno.

\section{REAVALIAÇÃO PÓS-PARTO}

A mulher com DMG deve ser reavaliada com teste de tolerância à glicose com sobrecarga de 75 gr no primeiro retorno pós-parto (seis semanas), com a finalidade de detecção daqueles casos em que algum grau de intolerância à glicose tenha persistido.

Deve-se lembrar que mesmo aquelas mulheres, cuja reavaliação foi normal, possuem um risco aumentado de desenvolvimento posterior de diabetes mellitus $^{(11,12,13)}$. Devem ser orientadas, portanto, quanto à necessidade de controle de peso, através da adoção de hábitos alimentares saudáveis e atividade física regular.

\section{GRAVIDEZ EM DIABÉTICAS COM DIAGNÓSTICO PRÉVIO}

O tratamento e monitorização das pacientes com diabetes mellitus antes da gestação é semelhante ao descrito anteriormente para o DMG.

Nesse grupo, um aspecto fundamental a ser abordado é a programação da gestação. A orientação 
quanto a isso deve ser realizada precocemente, no seguimento da mulher diabética em idade fértil. Deve-se buscar um controle adequado no período pré-concepção, já que há uma correlação bem definida de malformações fetais e hiperglicemia durante a embriogênese ${ }^{(5,23)}$.

Nas pacientes com DM2, os antidiabéticos orais devem ser suspensos e substituídos por insulina. Como no DMG, deve-se dar preferência à utilização de insulinas humanas ou purificadas.

A avaliação de comorbidades e complicações crônicas, principalmente as microangiopáticas, é ainda mais importante, nesse grupo, devido à possibilidade de piora durante a gestação ${ }^{(5,24)}$.

$\mathrm{O}$ manejo dessas pacientes, com DM prévio à gestação no período do parto, é semelhante ao daquelas com DMG. Após o parto, a necessidade de insuli- na diminui. Com isso, no puerpério imediato, a dose deve ser reduzida para a metade daquela administrada no dia anterior ao parto, utilizando-se insulina de ação rápida, conforme o perfil glicêmico, como descrito anteriormente para DMG. Após a alimentação ser regularizada, a depender do controle glicêmico, tanto as diabéticas do tipo 2, controladas antes da gravidez somente com dieta ou com antidiabéticos orais, quanto aquelas que usavam insulina antes da gestação devem retornar aos esquemas anteriores.

\section{AGRADECIMENTOS}

Agradecemos ao Dr. Wagner Lúcio Gueleri, Coordenador do Programa da Saúde da Mulher, da Secretaria Municipal da Saúde de Ribeirão Preto, e à Sra. Cláudia Siqueira Vassimon, pela colaboração.

MONTENEGRO JR RM; PACCOLA GMGF; FOSS MC; TORQUATO MTCG; YANO RK; MAUAD FILHO F; NOGUEIRA AA; BEREZOWSKI AT \& DUARTE G. Protocol for detection, diagnosis and treatment of diabetes mellitus during pregnancy. Medicina, Ribeirão Preto, 33: 520-527, oct.dec. 2000.

ABSTRACT: The authors present the approach to pregnant woman for screening and diagnosis of gestational diabetes mellitus (GDM) and treatment of the diabetes mellitus during pregnancy, established as routine by the Sector of High Risk Pregnancy of the Department and Gynecology and Obstetric and Division of Endocrinology and Metabolism of Medicine School of Ribeirão Preto - University of São Paulo and it was assumed as a routine pre-natal evaluation for screening and diagnosis of GDM at Ribeirão Preto Health Secretary's Office. This protocol was based on the recommendations of the World Health Organization and the American Diabetes Organization.

UNITERMS: Pregnancy in Diabetes. Diabetes, Gestational. Diagnosis. Clinical Protocols.

\section{REFERÊNCIAS BIBLIOGRÁFICAS}

1 - KITZMILLER JL; CLOHERTY JP; YOUNGER MD; TABATABAII A; ROTHCHILD SB; SOSENKO I; EPSTEIN MF; SINGH S \& NEFF RK. Diabetic pregnancy and perinatal morbidity. Am J Obstet Gynecol 131: 560-580, 1978.

2- WIDNESS JA; COWETT RM; COUSTAN DR; CARPENTER MW \& $\mathrm{OH}$ W. Neonatal morbidities in infants of mothers with glucose intolerance in pregnancy. Diabetes 34: 61-65, 1985. Suppl 2.

3 - PIMENTA WP; CUNHA SP \& FOSS MC. Gravidez e diabetes mellitus - experiência do período de 1980 a 1985 no Hospital das Clínicas da Faculdade de Medicina de Ribeirão Preto, Universidade de São Paulo. Rev Bras Ginecol Obstet 3: 54-61, 1988.

4- PACCOLAGMGF; TORQUATO MTG; BAIMA FILHO J; DUARTE
G \& FOSS MC. Diabetes Mellitus e gravidez: acompanhamento de 89 gestações de 1986 a 1991 no Hospital das Clínicas da Faculdade de Medicina de Ribeirão Preto - USP, Arq Bras Endocrinol Metab 39: 26-31, 1995.

5 - BEVIER WC; JOVANOVIC-PETERSON L \& PETERSON CM. Pancreatic disorders of pregnancy. Endocrinol Metab Clin North Am 24: 103-138, 1995.

6 - RUDGE MV; CALDERON IM; RAMOS MD; ABBADE JF \& RUGOLO LM. Perinatal outcome of pregnancies complicated by diabetes and by maternal daily hyperglycemia not related to diabetes a retrospective 10 years analysis. Gynecol Obstet Invest 50: 108-112, 2000

7 - THE EXPERT COMMITTEE ON THE DIAGNOSIS AND CLASSIFICATION OF DIABETES MELLITUS. AMERICAN DIABETES ASSOCIATION. Report of the Expert Committee on the Diagnosis and Classification of Diabetes Mellitus. Diabetes Care 20: 1183-1197, 1997. 
8- METZGER BE \& COUSTAN DR. Proceedings of the Fourth International Workshop-Conference on Gestational Diabetes Mellitus. Diabetes Care 21: B1- B167, 1998. Suppl 2

9 - COUSTAN DR \& IMARAH J. Prophyilactic insulin treatment of gestational diabetes reduces the incidence of macrossomia, operative delivery, and birth trauma. Am J Obstet Gynecol 150: 836-842, 1984.

10 - GABBE SG; MESTMAN JG; FREEMAN RK; ANDERSON GV \& LOWENSON RI. Management and outcome of class A diabetes mellitus. Am J Obstet Gynecol 127: 465-469, 1977.

11 - CATALANO PM; VARGO KM; BERNSTEIN IM \& AMINI SB. Incidence and risk factors associated with abnormal postpartum glucose tolerance in women with gestational diabetes. Am J Obstet Gynecol 165: 914-916, 1991.

12 - KJOS SL; BUCHANAN TA; GREENSPOON JS; MONTORO M; BERNSTEIN GS \& MESTMAN MD. Gestational diabetes mellitus: the prevalence of glucose intolerance and diabetes mellitus in the first 2 months postpartum. Am J Obstet Gynecol 163: 93-98, 1990.

13 - O'SULLIVAN JB. Diabetes after GDM. Diabetes 40: 131135, 1991. Suppl 2.

14 - COMBS CA; GUNDERSON E; KITZMILLER JL; GAVIN LA \& MAIN EK. Relationship of fetal macrosomia to maternal postprandial glucose control during pregnancy. Diabetes Care 15: 1251-1257, 1992.

15 - JOVANOVIC-PETERSON L, BEVIER W \& PETERSON CM. The Santa Barbara County Health Care Services Program: birthweight change concomitant with screening and treatment of glucose-intolerance of pregnancy: a potential costeffective intervention. Am J Perinatol 14: 221-228, 1997.

16 - ALBERTI KG \& ZIMMET PZ. Definition, diagnosis and classification of diabetes mellitus and its complications. Part 1: diagnosis and classification of diabetes mellitus provisional report of a WHO consultation. Diabet Med 15: 539553,1998 .
17 - AMERICAN DIABETES ASSOCIATION. Clinical Practice Recomendations 2000: Gestational Diabetes Mellitus. Diabetes Care 23: S77-S79, 2000. Suppl 1.

18 - DANILENKO-DIXON DR; VAN WINTER JT; NELSON RL \& OGBURN PL JR. Universal versus selective gestational diabetes screening: Application of 1997 American Diabetes Association recommendations. Am J Obstet Gynecol 181: 798-802, 1999.

19 - CHRISTESEN HBT \& MELANDER A. Prolongued elimination of tolbutamide in a premature newborn with hyperinsulinaemic hypoglycaemia. Eur J Endocrinol 138: 698-701, 1998.

20 - MONTENEGRO JR. RM; PACCOLA GMFG; FARIA CM; CUNHA SP \& FOSS MC. The use of sulfonylureas by pregnant women and fetal malformations. Rev Bras Ginecol Obstet 21: 393-397, 1999.

21 - BARBOSA FB; GRAVENA C; MATHIAS P \& MOURA AS. Blockade of the 32P phosphate flush of pancreaticcells from adult rats who received a low-protein diet during early lactation. Braz J Med Biol Res 26: 1355-1358, 1993.

22 - GIAVINI E; BROCCIAML; PRATI M \& DOMENICO ROVERSI G. Diet composition modifies embryotoxic effects induced by experimental diabetes in rats. Biol Neonate 59: 278-286, 1991.

23 - KITZMILLER JL; GAVIN LA; GIN GD; JOVANOVIC-PETERSON L; MAIN EK \& ZIGRANG WD. Preconception care of diabetes. Glycemic control prevents congenital malformations. JAMA 265: 731-736, 1991.

24 - GABBE SG \& LANDON MB. Diabetes mellitus and pregnancy. Adv Endocrinol Metab 5: 47-74, 1994.

Recebido para publicação em 08/08/2000

Aprovado para publicação em 30/11/2000 